\title{
Non- linear stability of triangular librations points in circular restricted three body under radiating and oblate primaries in presence of resonance
}

\author{
A Narayan $^{1 *}$, Nutan Singh ${ }^{2}$ \\ ${ }^{1}$ Department of Mathematics, Bhilai Institute of Technology, Durg, 491001, India \\ ${ }^{2}$ Department of Mathematics, Rungta College of Engg. and Technology \\ *Corresponding author E-mail:ashutoshmaths.narayan@gmail.com
}

\begin{abstract}
The nonlinear stability of the triangular librations points is studied in the presence resonance considering both the primaries as radiating and oblate. The study is carried out for various values of radiation pressure and oblateness parameter in general and binary systems in particular. It is found that the normal forms of the Hamiltonian contains both the resonance cases; $\omega_{1}=2 \omega_{2}$ and $\omega_{1}=3 \omega_{2}$. The case $\omega_{1}=\omega_{2}$ corresponds to the boundary region of the stability for the system.It is investigated that for $\mu \leq \mu_{c}=0.0385209$; the motion is unstable for third order resonance but stable for fourth order resonance.
\end{abstract}

Keywords:ER3BP, Hamiltonian Functions, Triangular Libration Points, Resonance.

\section{Introduction}

The three body problem deals with three spherical masses which interact with each other through the gravitational interactions described by Newton's theory of gravity, and no restrictions are imposed on initial positions and velocities. In restricted three body problem, the two primaries have dominant masses and move around their common centre of mass; however, the third mass is very-very small and its gravitational influence on the primaries is negligible. In celestial mechanics the bodies are not strictly spherical but are regarded as spheroids with small degree of oblateness. Manju and Choudhary (1985) studied the restricted problem of three bodiesby taking one of the bodies as radiating focusing on thenonlinear stability of the triangular libration points.Kumar and Choudhary $(1987,1988)$ investigated the stability of the triangular libration points for non-resonance as well as resonance case; taking both the bodies as radiating in circular restricted three body problems in presence of the third and fourth order resonance. Subba Rao and Sharma (1997) investigated the nonlinear stability of the triangular points by taking bigger primary as oblate and discussed three different ranges for critical values of mass ratios. Later on the study was extended by Markeev (2005), where the stability was studied focusing on the parametric resonance.

Many authors such as Danby (1964), Bennet (1965), Szebehely (1967) Markeev (1978), SelaruCucu-Dumitrescu (1995), Halan and Rana (2001) studied the behavior of equilibrium points in detail. The influence of the eccentricity of the orbits of the primaries with or without radiation pressure on the existence and stability of the equilibrium points was investigatedby Gyorgrey ( 1985), Grebenikov (1964), Kumar and Choudhary (1990), Markeellos (1992), Shaoo and Ishwar (2000), Roberts (2002), Floria (2004), Zimvoschikov and Thakai (2004), Ammar (2008), Erdi
(2009), Kumar and Ishwar (2011).Singh and Umar (2012a), (2012b), Usha et. al. (2014), Narayan and Singh (2014 a, b, c,). In the present paper an attempt has been made to analyze the existence of resonance and to study the nonlinear stability of infinitesimal in elliptical restricted three body problem near the resonance frequency satisfying $\omega_{1}=\omega_{2}, \omega_{1}=2 \omega_{2}, \omega_{1}=3 \omega_{2}$ in special case when $e=0$.The study is conducted by considering both the primaries as radiating and oblate under the presence of resonance. This model can be easily applied for the exoplanets, Trojan asteroids and for binary systems (Achird, Luyten, $\alpha$ Cen AB, kruger$60, \mathrm{Xi}$ - Bootis).

This paper is organized as follows:

Section 1 gives introduction; section 2 provides the equations of motion; while section 3 deals with characteristics roots and first order stability of the triangular equilibrium points. Existence of resonance and Normalisation for the cases $\omega_{1}=2 \omega_{2}$ and $\omega_{1}=3 \omega_{2}$ is given in section 4 . Section 5 and 6 focusses on Stability in the resonance case $\omega_{1}=2 \omega_{2}$ and $\omega_{1}=3 \omega_{2}$ respectively. Finally, discussion and conclusion are drawn in sections 7.

\section{Equations of motion}

The differential equations of the motion of the infinitesimal mass in ellipticalrestricted three body problem under radiating primaries in pulsating system is given by [Narayan and Shrivastava (2014)] as:

$$
\begin{aligned}
& x^{\prime \prime}-2 y^{\prime}=\varphi \Omega_{x} ; \\
& y^{\prime \prime}-2 x^{\prime}=\varphi \Omega_{y}
\end{aligned}
$$


Where

$$
\begin{aligned}
& \Omega= \frac{x^{2}+y^{2}}{2}+\frac{1}{1+3\left(\frac{A_{1}+A_{2}}{2}\right)} \times \\
& {\left[\begin{array}{l}
\frac{(1-\mu) Q_{1}}{r_{1}}+\frac{\mu Q_{2}}{r_{2}}+ \\
\left.\frac{(1-\mu) Q_{1} A_{1}}{2 r_{1}^{3}}+\frac{\mu A_{2} Q_{2}}{2 r_{2}^{3}}\right]
\end{array}\right] } \\
& r_{1}^{2}=(x+\mu)^{2}+y^{2} \\
& r_{2}^{2}=(x-1+\mu)^{2}+y^{2}
\end{aligned}
$$

And

$$
\varphi=\frac{1}{(1+e \cos \mathrm{f})}
$$

$A_{1}, A_{2}$ Are the oblateness parameter, $Q_{1}, Q_{2}$ Are the radiation pressure and $f$ is a true anomaly of the primaries, $e$ the eccentricity of the orbit and $\mu$ the mass ratio.

The coordinates of the triangular equilibrium points $\mathrm{L}_{4}$ and $\mathrm{L}_{5}$ are given by: [Narayan andShrivastava (2014)]

$$
\begin{aligned}
x_{0} & =\frac{1}{2}-\mu+\frac{A_{1}}{2}-\frac{A_{2}}{2}+\frac{\beta_{2}}{3}-\frac{\beta_{1}}{3} \\
- & \frac{1}{2} A_{1} \beta_{1}+\frac{1}{2} A_{2} \beta_{2} ; \\
y_{o} & = \pm \frac{\sqrt{3}}{2}\left[\begin{array}{l}
1-\frac{A_{1}}{3}-\frac{A_{2}}{3}-\frac{2 \beta_{1}}{9}-\frac{2 \beta_{2}}{9} \\
-\frac{1}{3} A_{1} \beta_{1}-\frac{1}{3} A_{2} \beta_{2}
\end{array}\right] .
\end{aligned}
$$

\section{Characteristics roots and first order stabil- ity of the triangular equilibrium points}

The Hamiltonian as described by Narayan and Shrivastava (2014) is given by:

$$
\begin{aligned}
& H=\frac{p_{1}^{2}+p_{2}^{2}}{2}+\left(p_{1} q_{2}-p_{2} q_{1}\right)+ \\
& \frac{\left(q_{1}^{2}+q_{2}^{2}\right) e \cos f}{2(1+e \cos f)}-\frac{1}{(1+e \cos f)\left(1+3 \frac{A_{1}+A_{2}}{2}\right)} \times \\
& \left\{\left\{\frac{(1-\mu) Q_{1}}{r_{1}}+\frac{\mu Q_{2}}{r_{2}}+\frac{(1-\mu) Q_{1} A_{1}}{2 r_{1}^{3}}+\frac{\mu A_{2} Q_{2}}{2 r_{2}^{3}}\right\}\right\}
\end{aligned}
$$

For simplicity of calculations consider

$$
Q=1-\beta_{i}, i=1,2 \text { and } \beta_{i}<1
$$

Equating the coefficients of 2nd 3rd, 4th order we get:

$$
\begin{aligned}
& H_{2}=\frac{p_{1}{ }^{2}+p_{2}{ }^{2}}{2}+\left(P_{1} q_{2}-P_{2} q_{1}\right)+ \\
& e \cos f \frac{\left(q_{1}^{2}+q_{2}^{2}\right)}{2(1+e \cos \mathrm{f})} \\
& -\frac{1}{(1+e \cos f)\left\{1+3\left(\frac{A_{1}+A_{2}}{2}\right)\right\}} \times
\end{aligned}
$$

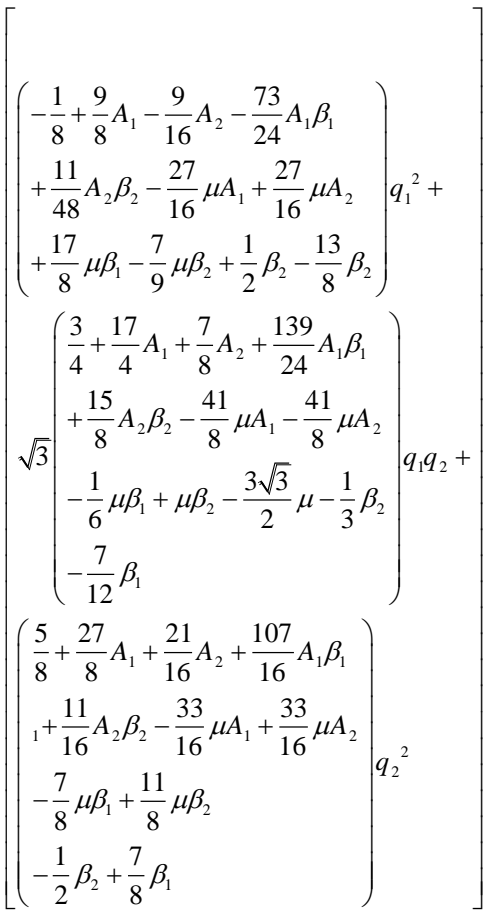

Equation (3.3) can be written as:

$$
H_{2}=\frac{p_{1}^{2}+p_{2}^{2}}{2}+\left(p_{1} q_{2}-p_{2} q_{1}\right)+
$$

$$
\frac{e \cos f}{2(1+e \cos f)\left\{1+3\left(\frac{A_{1}+A_{2}}{2}\right)\right\}}\left(q_{1}^{2}+q_{2}^{2}\right)
$$

$-H_{20} q_{1}^{2}-H_{11} q_{1} q_{2}-H_{02} q_{2}^{2}$

Provided,

$$
H_{20}=-\left(\begin{array}{c}
\frac{-1}{8}+\frac{9}{8} A_{1}-\frac{9}{16} A_{2}-\frac{73}{24} A_{1} \beta_{1} \\
+\frac{11}{48} A_{2} \beta_{2}-\frac{27}{16} \mu A_{1}+\frac{27}{16} \mu A_{2} \\
+\frac{17}{8} \mu \beta_{1}-\frac{7}{9} \mu \beta_{2}+\frac{\beta_{2}}{2}-\frac{13 \beta_{1}}{8}
\end{array}\right)
$$

$$
H_{11}=\sqrt{3}\left(\begin{array}{l}
\frac{3}{4}+\frac{17}{4} A_{1}+\frac{7}{8} A_{2}+\frac{139}{24} A_{1} \beta_{1} \\
+\frac{15}{8} A_{2} \beta_{2}-\frac{41}{8} \mu A_{1}-\frac{41}{8} \mu A_{2} \\
-\frac{1}{6} \mu \beta_{1}+\mu \beta_{2}-\frac{3 \mu}{2}-\frac{\beta_{2}}{3}-\frac{7 \beta_{1}}{12}
\end{array}\right)
$$

$$
H_{02}=\left(\begin{array}{c}
\frac{5}{8}+\frac{27}{8} A_{1}+\frac{21}{16} A_{2}+\frac{107}{16} A_{1} \beta_{1} \\
+\frac{11}{16} A_{2} \beta_{2}-\frac{33}{8} \mu A_{1}+\frac{33}{16} \mu A_{2} \\
-\frac{7}{8} \mu \beta_{1}+\frac{11}{8} \mu \beta_{2}-\frac{\beta_{2}}{2}+\frac{7 \beta_{1}}{8}
\end{array}\right)
$$




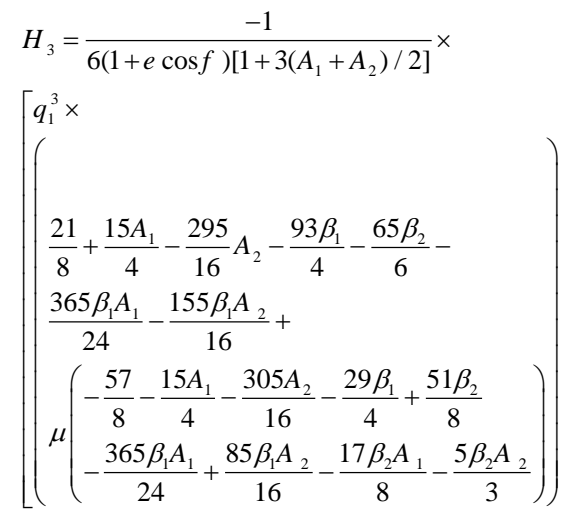

$+3 q_{1}^{2} q_{2} \times$

$$
\left(\begin{array}{l}
\frac{-3}{8}+\frac{21 A_{1}}{8}-\frac{61}{8} A_{2}-\frac{113 \beta_{1}}{24}+\frac{7 \beta_{2}}{4} \\
-\frac{53 \beta_{1} A_{1}}{24}+\frac{61 \beta_{1} A_{2}}{8}+ \\
\mu \sqrt{3}\left(\begin{array}{l}
\frac{17 A_{1}}{16}+\frac{9 A_{2}}{16}+\frac{47 \beta_{1}}{6}-\frac{13 \beta_{2}}{4}-\frac{53 \beta_{1} A_{1}}{24} \\
-\frac{113 \beta_{1} A_{2}}{24}-\frac{91 \beta_{2} A_{1}}{16}-\frac{\beta_{2} A_{2}}{48}
\end{array}\right)
\end{array}\right)
$$

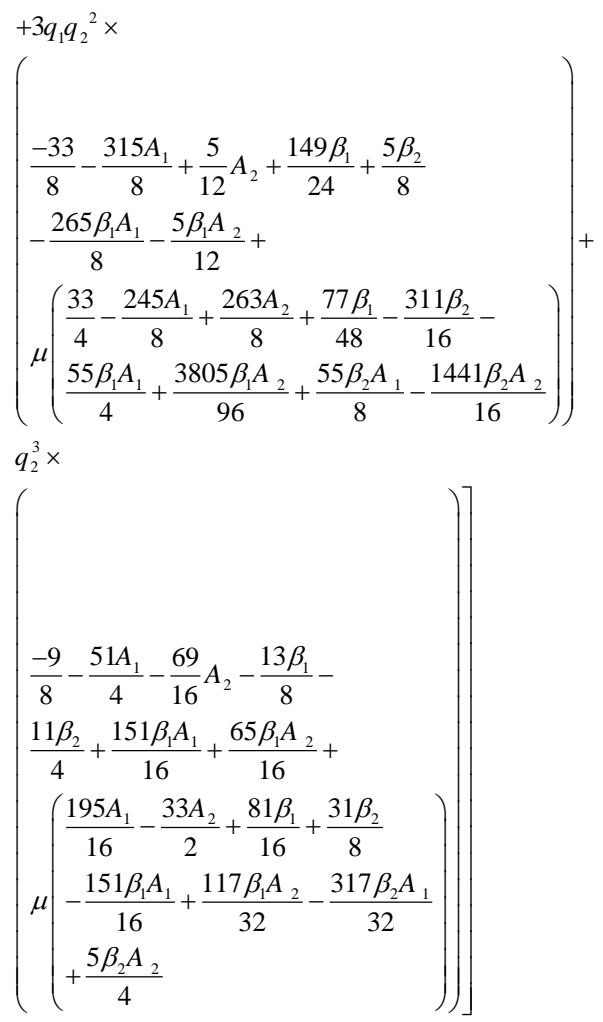

$$
H_{4}=\frac{-1}{24(1+e \cos f)} \times\left[1+3\left(A_{1}+A_{2}\right) / 2\right] \times
$$$$
\left\lceil q_{1}^{4} \times\right.
$$$$
\frac{-111}{16}-\frac{1155 A_{1}}{16}+\frac{9815}{16} A_{2}+\frac{397 \beta_{1}}{2}
$$$$
-\frac{25 \beta_{2}}{2}-\frac{1235 \beta_{1} A_{1}}{16}-\frac{1185 \beta_{1} A_{2}}{2}
$$$$
+\mu\left(\begin{array}{l}
-\frac{1575}{16}-\frac{395 A_{1}}{48}-\frac{29545 A_{2}}{48}+\frac{9269 \beta_{1}}{16} \\
+\frac{439 \beta_{2}}{144}-\frac{1234 \beta_{1} A_{1}}{16}-\frac{6325 \beta_{1} A_{2}}{12}+ \\
\frac{6755 \beta_{2} A_{1}}{48}+\frac{605 \beta_{2} A_{2}}{48}
\end{array}\right)
$$

$$
\left[\begin{array}{l}
+4 \sqrt{3} q_{1}^{3} q_{2} \times \\
-75 \\
\frac{-281 A_{1}}{16}-\frac{21}{2} A_{2}+\frac{5 \beta_{1}}{16} \\
+\frac{21 \beta_{2}}{6}+\frac{351 \beta_{1} A_{1}}{16}+\frac{21 \beta_{1} A_{2}}{2} \\
\left(\begin{array}{l}
\frac{75}{8}+\frac{5669 A_{1}}{208}-\frac{117 A_{2}}{8}-\frac{235 \beta_{1}}{48} \\
-\frac{6121 \beta_{2}}{560}-\frac{7 \beta_{1} A_{1}}{2}+\frac{319 \beta_{1} A_{2}}{32}+ \\
\frac{2357 \beta_{2} A_{1}}{156}-\frac{83 \beta_{2} A_{2}}{4}
\end{array}\right)
\end{array}\right)
$$

$$
+\left(\begin{array}{l}
6 q_{1}^{2} q_{2}^{2} \times \\
+\left(\begin{array}{l}
\frac{123}{16}+\frac{4865 A_{1}}{48}-\frac{105}{8} A_{2}+\frac{235 \beta_{1}}{12} \\
+\frac{75 \beta_{2}}{12}-\frac{4295 \beta_{1} A_{1}}{48}+\frac{105 \beta_{1} A_{2}}{8} \\
+\mu\left(\begin{array}{l}
-\frac{123}{8}-\frac{5735 A_{1}}{48}+\frac{1601 A_{2}}{16}-\frac{421 \beta_{1}}{48} \\
-\frac{31 \beta_{2}}{45 \beta_{2} A_{1}}
\end{array}\right)-\frac{539 \beta_{2} A_{1}}{4}+\frac{245 \beta_{1} A_{2}}{32}+
\end{array}\right)
\end{array}\right)
$$




$$
\left[\begin{array}{l}
+4 \sqrt{3} q_{1} q_{2}{ }^{3} \times \\
\frac{135}{16}+\frac{1531 A_{1}}{16}+\frac{15}{4} A_{2}-\frac{69 \beta_{1}}{16} \\
-\frac{21 \beta_{2}}{5}+\frac{23 \beta_{1} A_{1}}{2}-\frac{15 \beta_{1} A_{2}}{4} \\
+\mu\left(\begin{array}{l}
-\frac{135}{8}-\frac{337 A_{1}}{2}-\frac{8997 A_{2}}{112} \\
+\frac{259 \beta_{1}}{16}+\frac{293 \beta_{2}}{40}+\frac{1391 \beta_{1} A_{1}}{16} \\
-\frac{3005 \beta_{1} A_{2}}{32}+\frac{1315 \beta_{2} A_{1}}{116}-\frac{175 \beta_{2} A_{2}}{48}
\end{array}\right)
\end{array}\right)
$$

$$
+q_{2}^{4}\left(\begin{array}{c}
\frac{9}{16}+\frac{1125 A_{1}}{16}-\frac{1575}{32} A_{2}+\frac{1781 \beta_{1}}{16} \\
-\frac{45 \beta_{2}}{2}-\frac{1045 \beta_{1} A_{1}}{8}+\frac{1575 \beta_{1} A_{2}}{32} \\
+\mu\left(\begin{array}{l}
-\frac{495 A_{1}}{8}-\frac{3167 A_{2}}{32}-\frac{1917 \beta_{1}}{16} \\
+26 \beta_{2}+\frac{1045 \beta_{1} A_{1}}{8}-\frac{14765 \beta_{1} A_{2}}{96}- \\
\frac{1325 \beta_{2} A_{1}}{32}-\frac{6775 \beta_{2} A_{2}}{48}
\end{array}\right)
\end{array}\right)
$$

\section{Existence of resonance}

As discussed by Liapunov's (1956), the equilibrium points are stable if $\mathrm{H}_{2}$ is of positive definite form; otherwise stability can be investigated by Arnold's theorem [1963(a, b)] .In this theorem $\mathrm{H}_{2}$ is normalized using linear transformation of variables as given by Markeev (1978). Restricting to $\mathrm{H}_{2}$ alone the characteristics equation takes the following form:

$$
\left|\begin{array}{cc}
\lambda^{2}-\left(1-2 H_{20}\right) & -2 \lambda-H_{11} \\
2 \lambda-H_{11} & \lambda^{2}-\left(1+2 H_{02}\right)
\end{array}\right|=0
$$

Where $H_{20}, H_{11}, H_{02}$ are given by equation [3.5-3.7]. After further calculations the characteristics equation reduces to the form:

$$
\begin{aligned}
& \lambda^{4}+\lambda^{2}+\left\{\frac{27 \mu(1-\mu)}{4}\right\} \times \\
& \left\{1-\frac{125}{6} A_{1}+\frac{40}{3} A_{2}+\frac{5}{6} \beta_{1}-\frac{35}{18} \beta_{2}\right\}=0
\end{aligned}
$$

If $\omega_{1}$ and $\omega_{2}$ be the frequencies then the equation can be written as:

$$
\begin{aligned}
& \omega^{4}-\omega^{2}+\left\{\frac{27 \mu(1-\mu)}{4}\right\} \times \\
& \left\{1-\frac{125}{6} A_{1}+\frac{40}{3} A_{2}+\frac{5}{6} \beta_{1}-\frac{35}{18} \beta_{2}\right\}=0
\end{aligned}
$$

The roots of equation (4.3) are given as:

$$
\begin{aligned}
& \left.\omega_{1,2}^{2}=\frac{1}{2}\left[1+\left\{\begin{array}{l}
1-27 \mu(1-\mu) \times \\
\left.1-\frac{125}{6} A_{1}+\frac{40}{3} A_{2}+\right) \\
\frac{5}{6} \beta_{1}-\frac{35}{18} \beta_{2}
\end{array}\right)\right\}^{1 / 2}\right] \\
& \omega_{3,4}^{2}=\frac{1}{2}\left[1-\left\{\begin{array}{l}
1-27 \mu(1-\mu) \times \\
\left(1-\frac{125}{6} A_{1}+\frac{40}{3} A_{2}+\right. \\
\frac{5}{6} \beta_{1}-\frac{35}{18} \beta_{2}
\end{array}\right)\right\}
\end{aligned}
$$

Figs 1-5 show the value of $\omega_{1}$ and $\omega_{2}$ for different values of $\mu$.

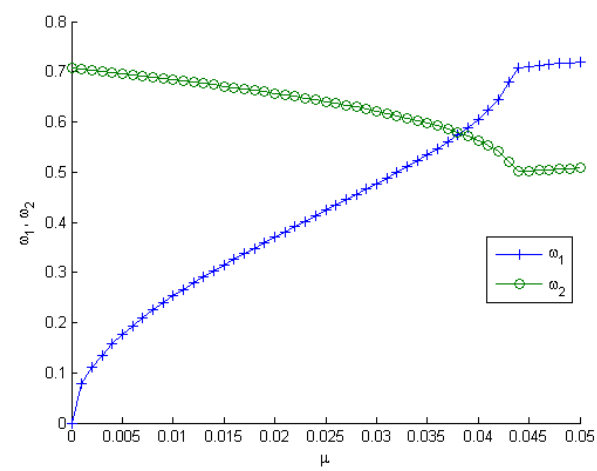

Fig.1: Correlation between $\mu$ and $\omega_{1}$ and $\omega_{2}$ with $\beta_{1}=0.01, \beta_{2}=0.02$, $\mathrm{A}_{1}=0.01, \mathrm{~A}_{2}=0.02$

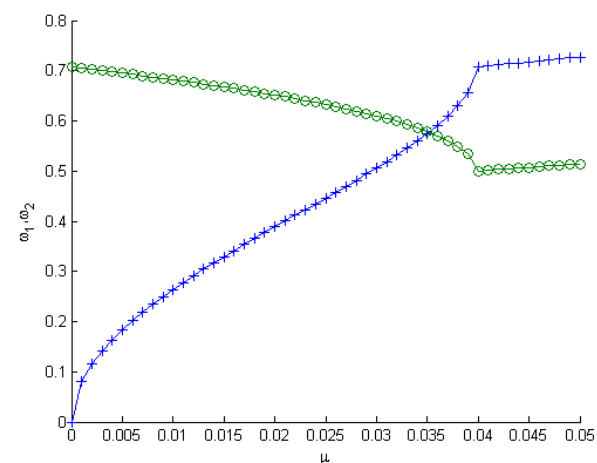

Fig.2: Correlation between $\mu$ and $\omega_{1}$ and $\omega_{2}$ with $\beta_{1}=0.01, \beta_{2}=0.02$, $\mathrm{A}_{1}=0.02, \mathrm{~A}_{2}=0.03$.

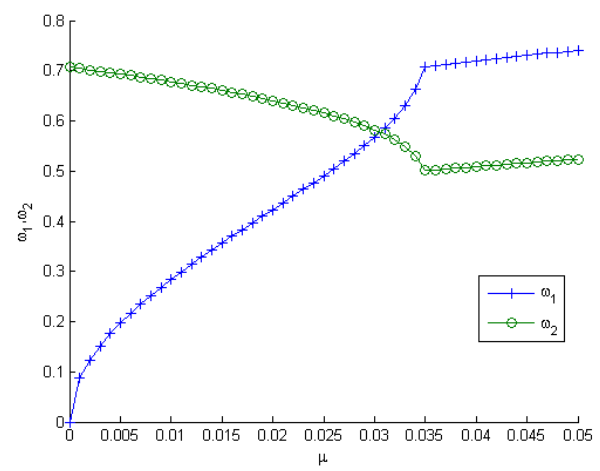

Fig. 3: Correlation between $\mu$ and $\omega_{1}$ and $\omega_{2}$ with $\beta_{1}=0.01, \beta_{2}=0.02$, $\mathrm{A}_{1}=0.02, \mathrm{~A}_{2}=0.035$. 


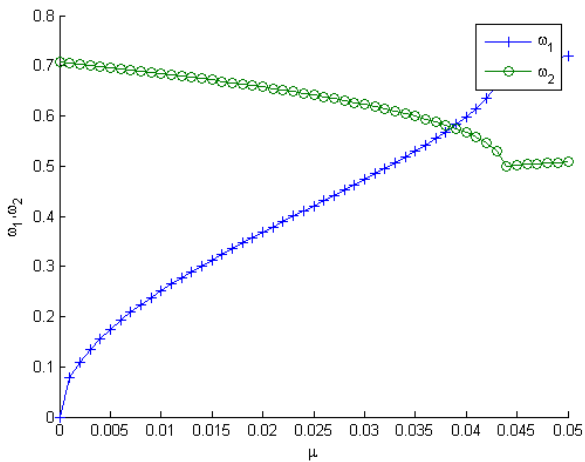

Fig. 4: Correlation between $\mu$ and $\omega_{1}$ and $\omega_{2}$ with $\beta_{1}=0.02, \beta_{2}=0.02$, $\mathrm{A}_{1}=0.01, \mathrm{~A}_{2}=0.02$.

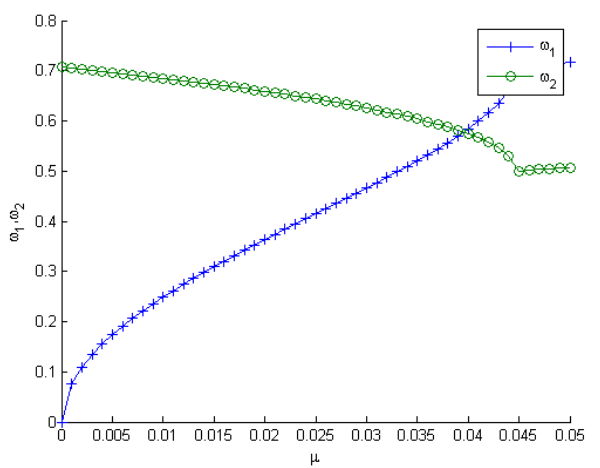

Fig. 5: Correlation between $\mu$ and $\omega_{1}$ and $\omega_{2}$ with $\beta_{1}=0.02, \beta_{2}=0.03$, $\mathrm{A}_{1}=0.01, \mathrm{~A}_{2}=0.02$.

It is seen from Figs 1-5 that for various value of radiation pressure and varying oblateness $\omega_{1}$ decreases with increasing $\mu$ whereas $\omega_{2}$ increases, and becomes equal for $\mu=\mu_{c}$.

Let us consider the following cases:

Case1. When $\omega_{1}=\omega_{2}$

$\lambda_{1,2}^{2}=\lambda_{3,4}^{2}$

Then we have:

$$
1-27 \mu(1-\mu)\left(\begin{array}{l}
1-\frac{125}{6} A_{1}+\frac{40}{3} A_{2} \\
+\frac{5}{6} \beta_{1}-\frac{35}{18} \beta_{2}
\end{array}\right) \geq 0
$$

If the equality holds then we have:

$$
\begin{aligned}
& 27\left(1-\frac{125}{6} A_{1}+\frac{40}{3} A_{2}+\frac{5}{6} \beta_{1}-\frac{35}{18} \beta_{2}\right) \mu^{2}- \\
& 27\left(1-\frac{125}{6} A_{1}+\frac{40}{3} A_{2}+\frac{5}{6} \beta_{1}-\frac{35}{18} \beta_{2}\right) \mu+1=0
\end{aligned}
$$

i.e.

$$
\mu=\frac{9 \pm \sqrt{69}\left(\begin{array}{l}
1-\frac{125}{69} A_{1}+\frac{80}{69} A_{2}+ \\
\frac{5}{69} \beta_{1}-\frac{35}{207} \beta_{2}
\end{array}\right)}{18}
$$

Since $\mu \leq \frac{1}{2}$, the positive sign is inadmissible. Hence the region of stability in first approximation can be written as $0<\mu<\frac{\left\{9-\sqrt{69}\left(\begin{array}{l}1-\frac{125}{69} A_{1}+\frac{80}{69} A_{2}+ \\ \frac{5}{69} \beta_{1}-\frac{35}{207} \beta_{2}\end{array}\right)\right\}}{18}$

$\mu_{\mathrm{c}}=0.0385208965+0.83601 A_{1}+$

$0.535048 A_{2}-0.0334405 \beta_{1}+0.0780279 \beta_{2}$

The critical value $\left(\mu_{c}\right)$ usually corresponds to the boundary of the region of stability of the linear system.

Case2. When $\omega_{1}=2 \omega_{2}$

i.e. $\lambda_{1,2}^{2}=4 \lambda_{3,4}^{2}$

Solving for $\mu$ the resonance value is obtained as:

$$
\begin{aligned}
& \mu_{\mathrm{c} 1}=\frac{1}{2} \pm \\
& \sqrt{\frac{1}{4}-\frac{16}{675\left(1-\frac{125 A_{1}}{6}+\frac{40 A_{2}}{3}+\frac{5 \beta_{1}}{6}-\frac{35 \beta_{2}}{18}\right)}}
\end{aligned}
$$

Case 3 . When $\omega_{1}=3 \omega_{2}$

i.e. $\lambda_{1,2}^{2}=9 \lambda_{3,4}^{2}$

The value of $\mu$ is given as:

$\mu_{\mathrm{c} 2}=\frac{1}{2} \pm$

$\sqrt{\frac{1}{4}-\frac{9}{675\left(1-\frac{125 A_{1}}{6}+\frac{40 A_{2}}{3}+\frac{5 \beta_{1}}{6}-\frac{35 \beta_{2}}{18}\right)}}$

The values of $\mu_{\mathrm{c} 1}$ and $\mu_{\mathrm{c} 2}$ are plotted for various values of oblateness parameters which is shown in Figs 6-15.It is clear from these figs. that resonance condition exists for cases $\omega_{1}=2 \omega_{2}$ and $\omega_{1}=$ $3 \omega_{2}$ for different values of oblateness and radiation pressure.

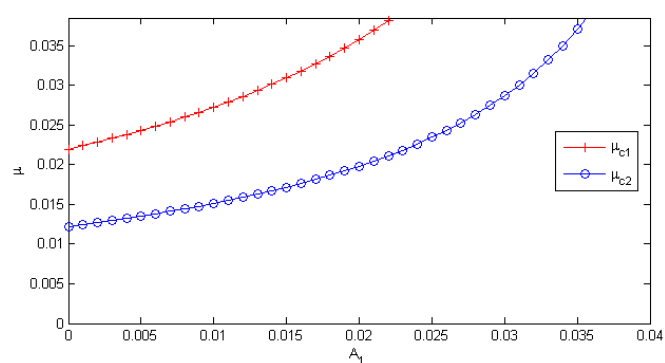

Fig. 6: $\mu_{C 1}, \mu_{C 2}$ vs $A_{1}$ when $A_{2}=0.01, \beta_{1}=0.01, \beta_{2}=0.02$.

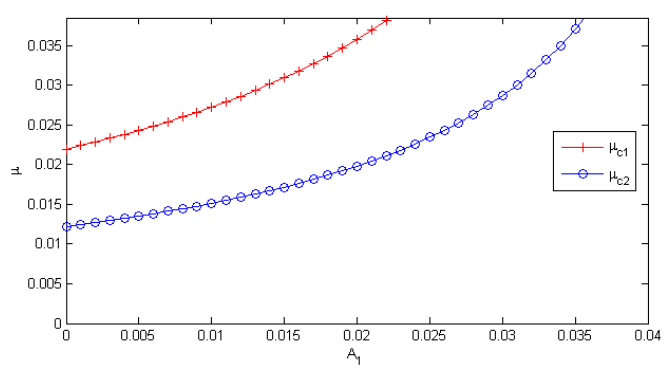

Fig. 7: $\mu_{\mathrm{C} 1}, \mu_{\mathrm{C} 2}$ vs $A_{1}$ when $A_{2}=0.02, \beta_{1}=0.01, \beta_{2}=0.02$. 


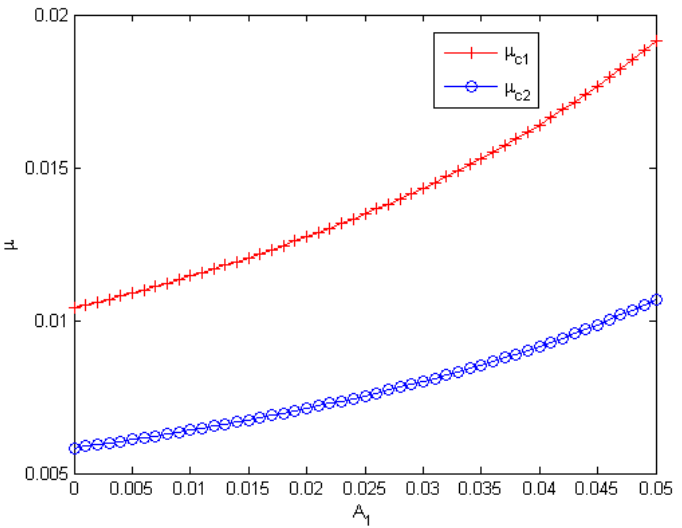

Fig. 8: $\mu_{\mathrm{C} 1}, \mu_{\mathrm{C} 2}$ vs $A_{1}$ when $A_{2}=0.1, \beta_{1}=0.01, \beta_{2}=0.02$.

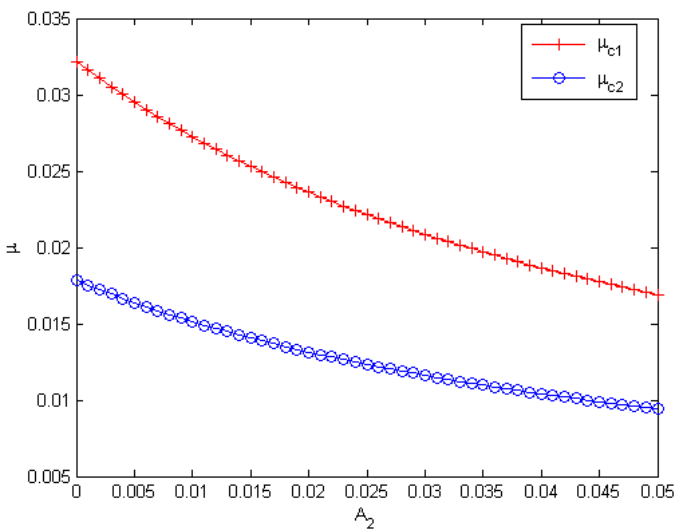

Fig. 9: $\mu_{\mathrm{C} 1}, \mu_{\mathrm{C} 2}$ vs $A_{2}$ when $A_{1}=0.01, \beta_{1}=0.01, \beta_{2}=0.02$.

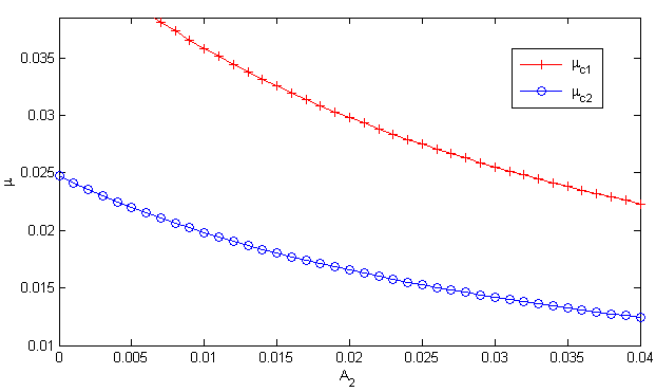

Fig. 10: $\mu_{C 1}, \mu_{C 2}$ vs $A_{2}$ when $A_{1}=0.02, \beta_{1}=0.01, \beta_{2}=0.02$.

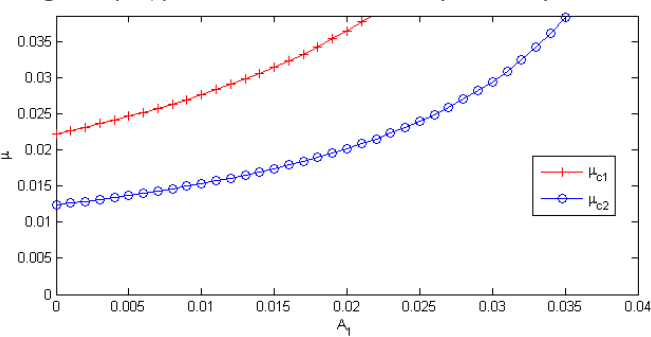

Fig.11: $\mu_{\mathrm{c} 1}, \mu_{\mathrm{c} 2}$ vs $\mathrm{A}_{1}$ when $\mathrm{A}_{2}=0.01, \beta_{1}=0.02, \beta_{2}=0.03$.

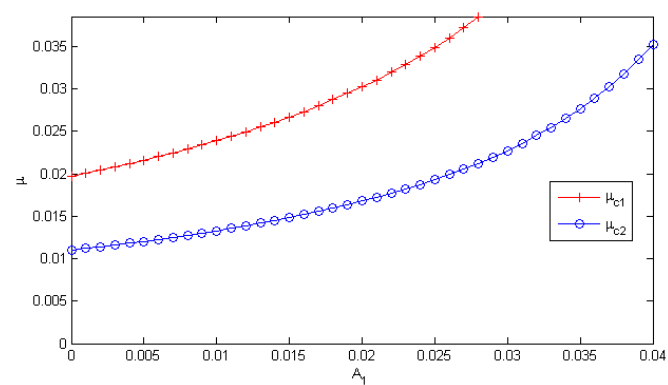

Fig. 12: $\mu_{\mathrm{C} 1}, \mu_{\mathrm{C} 2}$ Vs $A_{1}$ when $A_{2}=0.02, \beta_{1}=0.02, \beta_{2}=0.03$.

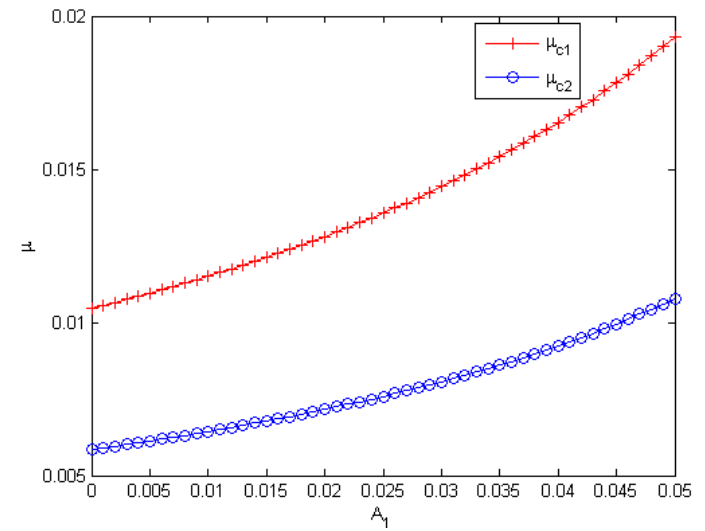

Fig .13: $\mu_{\mathrm{C} 1}, \mu_{\mathrm{C} 2}$ vs $\mathrm{A}_{1}$ when $\mathrm{A}_{2}=0.1, \beta_{1}=0.02, \beta_{2}=0.03$.

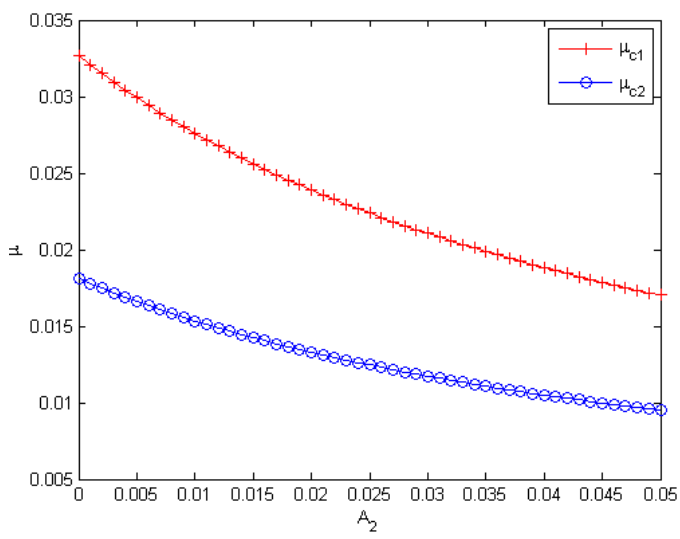

Fig .14: $\mu_{\mathrm{C} 1}, \mu_{\mathrm{C} 2}$ vs $A_{2}$ when $A_{1}=0.01, \beta_{1}=0.02, \beta_{2}=0.03$.

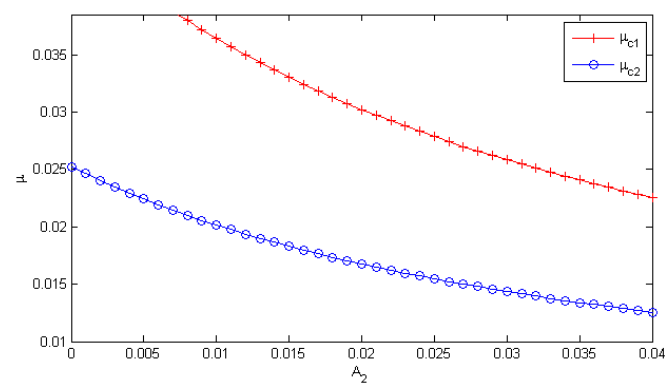

Fig .15: $\mu_{\mathrm{C} 1}, \mu_{\mathrm{C} 2}$ vs $A_{2}$ when $A_{1}=0.02, \beta_{1}=0.02, \beta_{2}=0.03$.

Further, in order to discuss the stability of the triangular points, the Hamiltonian $\mathrm{H}$ is normalized up to fourth order by using suitable canonical transformation as:

$\left(q_{1}, q_{2}, p_{1}, p_{2}\right)=\left(q_{1}^{\prime}, q_{2}^{\prime}, p_{1}{ }_{1}, p^{\prime}{ }_{2}\right) N$

Where,

$$
N=\left[\begin{array}{cccc}
a_{1} & a_{1} c_{1} & -a_{1} c_{1} & a_{1}\left(1-\omega_{1}^{2} b_{1}\right) \\
a_{2} & a_{2} c_{2} & -a_{2} c_{2} & a_{2}\left(1-\omega_{2}^{2} b_{2}\right) \\
0 & a_{1} b_{1} & a_{1}\left(1-b_{1}\right) & a_{1} c_{1} \\
0 & -a_{2} b_{2} & -a_{2}\left(1-b_{2}\right) & -a_{2} c_{2}
\end{array}\right]
$$

And

$a_{1}=\frac{1}{2}\left(\frac{2 l_{1}}{\omega_{1}^{2}-1 / 2}\right)^{1 / 2} ;$

$a_{2}=\frac{1}{2}\left(\frac{2 l_{2}}{\omega_{2}^{2}-1 / 2}\right)^{1 / 2}$

$l_{1}=1+\omega_{1}^{2}+2 H_{02} ; \quad l_{2}=1+\omega_{2}^{2}+2 H_{02}$ 


$$
\begin{aligned}
& b_{1}=\frac{2}{l_{1}} ; \quad b_{2}=\frac{2}{l_{2}} \\
& c_{1}=\frac{-H_{11}}{l_{1}} ; c_{2}=\frac{-H_{11}}{l_{2}}
\end{aligned}
$$

The transformation (4.12) reduces the Hamiltonian to the following form:

$$
\begin{aligned}
& H=\frac{1}{2}\left(p_{1}^{\prime 2}+\omega_{1}^{2} q_{1}^{\prime 2}\right)-\frac{1}{2}\left(p_{2}^{\prime 2}+\omega_{2}^{2} q_{2}^{2}\right) \\
& +\sum_{\alpha+\gamma=3}^{\infty} h_{\alpha_{1}, \alpha_{2} \beta_{1}, \beta_{2}} q_{1}^{\prime \alpha_{1}} q_{2}^{\prime \alpha_{2}} p_{1}^{\prime \gamma_{2}} p_{2}^{\prime \gamma_{2}}
\end{aligned}
$$

Where, $\alpha=\alpha_{1}+\alpha_{2} ; \gamma=\gamma_{1}+\gamma_{2}$

The coefficient of third and fourth order terms of $h_{\alpha_{1} \alpha_{2} \gamma_{1} \gamma_{2}}^{\prime}$ and $h_{\alpha_{1} \alpha_{2} \gamma_{1} \gamma_{2}}$ is given in appendices [see (a)].

Again using the BirKhoffs transformation the Hamiltonian given by (4.15) reduces to the following form:

$$
\begin{aligned}
& \bar{H}=i\left(\omega_{1} q_{1}{ }^{\prime \prime} p_{1}{ }^{\prime \prime}+\omega_{2} q_{2}{ }^{\prime \prime} p_{2}{ }^{\prime \prime}\right) \\
& +\sum_{\alpha+\gamma=3}^{\infty} h^{\prime}{ }_{\alpha_{1}, \alpha_{2} \gamma_{1}, \gamma_{2}} q_{1}^{\prime \prime \alpha_{1}} q_{2}{ }^{\prime \prime \alpha_{2}} p_{1}{ }^{\prime \gamma_{1}} p_{2}{ }^{\prime \prime} \gamma_{2}
\end{aligned}
$$

\section{Where}

$h^{\prime}=x_{\alpha_{1}, \alpha_{2}, \gamma_{1}, \gamma_{2}}+i y_{\alpha_{1}, \alpha_{2}, \gamma_{1}, \gamma_{2}}$

The values of $x_{\alpha_{1}, \alpha_{2}, \gamma_{1}, \gamma_{2}}$, and $y_{\alpha_{1}, \alpha_{2}, \gamma_{1}, \gamma_{2}}$ are given in appendices [see (b)].

The other coefficients of third order terms are given in the appendices $[$ see $(c)]$.

\section{Stability in the resonance case $\omega_{1}=2 \omega_{2}$}

In the resonance case $\omega_{1}=2 \omega_{2}$, using the Birkhoffs transformation

$$
\left(q{ }^{\prime}, p{ }_{j}\right) \rightarrow\left(Q_{j}, P_{j}\right)
$$

It is not possible to cancel whole $\bar{H}_{3}$ of the Hamiltonian $H$.In this case $\bar{H}_{3}$ retains two resonant terms with coefficients $h_{1002}^{\prime}$ and $h^{\prime}{ }_{0210}$. Thus the normalised form of the Hamiltonian is given as;

$\bar{H}=i\left(\omega_{1} Q_{1} P_{1}+\omega_{2} Q_{2} P_{2}\right)+h^{\prime}{ }_{1002} Q_{1} P_{2}^{2}+$

$h^{\prime}{ }_{0210} Q_{2}^{2} P_{1}+\ldots .$.
Applying two canonical transformations as:

$Q_{1}=\frac{1}{\left(\omega_{1}\right)^{1 / 2}}\left(Q_{1}^{0}-i P_{1}^{0}\right)$

$Q_{2}=\frac{1}{\left(\omega_{2}\right)^{1 / 2}}\left(i Q_{2}^{0}-P_{2}^{0}\right)$

$P_{1}=\frac{\left(\omega_{1}\right)^{1 / 2}}{2}\left(-i Q_{1}^{0}+P_{1}^{0}\right)$

$P_{2}=\frac{\left(\omega_{2}\right)^{1 / 2}}{2}\left(i Q_{2}^{0}-i P_{2}^{0}\right)$

And

$Q_{1}^{0}=\left(2 r_{1}\right)^{1 / 2} \sin \left(\varphi_{1}-\theta_{1}\right)$

$P_{1}^{0}=\left(2 r_{1}\right)^{1 / 2} \cos \left(\varphi_{1}-\theta_{1}\right) ; Q_{2}^{0}=\left(2 r_{2}\right)^{1 / 2} \sin \varphi_{2} ;$

$P_{2}^{0}=\left(2 r_{1}\right)^{1 / 2} \cos \varphi_{2}$

Where

$\sin \theta_{1}=\frac{y_{1002}}{\left(x_{1002}^{2}+y_{1002}^{2}\right)^{1 / 2}}$

$\cos \theta_{1}=\frac{x_{1002}}{\left(x_{1002}^{2}+y_{1002}^{2}\right)^{1 / 2}}$.

The Hamiltonian given by equation (5.1) is reduced to the following polar form as:

$$
\begin{aligned}
& \bar{H}=2 \omega_{2} r_{1}-\omega_{2} r_{2}-\sqrt{\left(x_{1002}^{2}+y_{1002}^{2}\right) \omega_{2} r_{2}} \times \\
& \sqrt{r_{1}} \sin \left(\varphi_{1}+\varphi_{2}\right)+o\left(\left(r_{1}+r_{2}\right)^{2}\right)
\end{aligned}
$$

The nonlinear stability of the equilibrium points is determined by Markeev theorem (1968). The equilibrium points are stable, if

$$
\left(x_{1002}^{2}+y_{1002}^{2}\right)=0 ; \text { and } c_{20}+2 c_{11}+4 c_{02} \neq 0
$$

\begin{tabular}{|c|c|c|c|c|c|c|c|c|}
\hline$\beta_{1}$ & $\beta_{2}$ & $\mathrm{~A}_{1}$ & $\mathrm{~A}_{2}$ & $\begin{array}{l}\left(x_{1002}^{2}+y_{1002}^{2}\right) \\
\times\left(10^{3}\right)\end{array}$ & $\begin{array}{l}c_{20}+2 c_{11}+4 c_{02} \\
\times\left(10^{4}\right)\end{array}$ & $\begin{array}{l}x_{1003}^{2}+y_{1003}^{2} \\
\times\left(10^{7}\right)\end{array}$ & $\begin{array}{l}c_{20}+3 c_{11}+9 c_{02} \\
\times\left(10^{5}\right)\end{array}$ & $\begin{array}{l}3 \omega_{2} \times \\
\left(x_{1003}^{2}+y_{1003}^{2}\right)^{1 / 2} \\
\times\left(10^{4}\right)\end{array}$ \\
\hline 0.01 & 0.01 & 0.01 & 0.02 & 2.2500 & 7.3266 & 5.2698 & 1.7226 & 1.4048 \\
\hline 0.01 & 0.01 & 0.02 & 0.03 & 2.1361 & 8.3939 & 5.7718 & 1.7957 & 1.4373 \\
\hline 0.01 & 0.01 & 0.03 & 0.035 & 1.7953 & 4.4397 & 6.7146 & 8.4798 & 1.4328 \\
\hline 0.01 & 0.02 & 0.01 & 0.02 & 1.9619 & 7.8852 & 4.8128 & 1.8300 & 1.3430 \\
\hline 0.01 & 0.02 & 0.02 & 0.03 & 1.8444 & 8.7172 & 5.1042 & 1.8582 & 1.3516 \\
\hline 0.01 & 0.02 & 0.03 & 0.035 & 1.5138 & 4.4578 & 5.3261 & 8.5033 & 0.3262 \\
\hline 0.01 & 0.03 & 0.01 & 0.02 & 1.6974 & 8.4615 & 4.4190 & 1.9420 & 0.2739 \\
\hline 0.01 & 0.03 & 0.02 & 0.03 & 1.5788 & 9.0441 & 4.5157 & 1.9222 & 0.3276 \\
\hline 0.01 & 0.03 & 0.03 & 0.035 & 1.2597 & 4.4679 & 4.1075 & 0.85130 & 0.3252 \\
\hline 0.01 & 0.04 & 0.01 & 0.02 & 1.4561 & 9.0566 & 4.0672 & 2.0590 & 0.2740 \\
\hline
\end{tabular}

are true simultaneously. From table (1) and (2) it is clear that for no values of the radiation pressure and oblateness parameters the expression $\left(x_{1002}^{2}+y_{1002}^{2}\right)$ vanishes. Hence it follows that the motion is unstable in the resonance case $\omega_{1}=2 \omega_{2}$ in general and for the binary system (Achird, Luyten, $\alpha$ Cen AB, kruger-60, Xi- Bootis), in particular.

Table 1: Values of $x_{1002}^{2}+y_{1002}^{2}, c_{20}+2 c_{11}+4 c_{02}, x_{1003}^{2}+y_{1003}^{2}, c_{20}+3 c_{11}+9 c_{02}$ and $3 \omega_{2}\left(x_{1003}^{2}+y_{1003}^{2}\right)^{1 / 2}$ for different Values of Radiation Pressure and Oblateness. 


\begin{tabular}{|c|c|c|c|c|c|c|c|c|}
\hline 0.01 & 0.04 & 0.02 & 0.03 & 1.3390 & 9.3749 & 3.9877 & 1.9876 & 0.3276 \\
\hline 0.01 & 0.04 & 0.03 & 0.035 & 1.0332 & 4.4687 & 3.0446 & .85066 & 0.3241 \\
\hline 0.02 & 0.01 & 0.01 & 0.02 & 2.7088 & 8.2907 & 6.8965 & 1.9799 & 0.2743 \\
\hline 0.02 & 0.02 & 0.02 & 0.03 & 2.2455 & 9.8846 & 6.8744 & 2.1239 & 0.3286 \\
\hline 0.02 & 0.03 & 0.03 & 0.035 & 1.5380 & 5.1843 & 5.1250 & .99226 & 0.3279 \\
\hline 0.02 & 0.04 & 0.01 & 0.02 & $1.8276 \mathrm{e}$ & 10.310 & 5.5136 & 2.3712 & 0.2746 \\
\hline 0.02 & 0.04 & 0.02 & 0.03 & 1.6797 & 10.658 & 5.4871 & 2.2749 & 0.3286 \\
\hline 0.02 & 0.05 & 0.03 & 0.035 & 1.2858 & 5.2009 & 2.8704 & .99480 & 0.3259 \\
\hline 0.03 & 0.01 & 0.01 & 0.02 & 3.2131 & 9.2630 & 9.1260 & 2.2585 & 0.2748 \\
\hline 0.03 & 0.03 & 0.03 & 0.035 & 1.8427 & 5.9342 & 6.4323 & 1.1436 & 0.3304 \\
\hline 0.04 & 0.01 & 0.01 & 0.02 & 3.7635 & 10.221 & 12.143 & 2.5579 & 0.2754 \\
\hline 0.04 & 0.02 & 0.02 & 0.03 & 3.1710 & 12.467 & 12.309 & 2.7359 & 0.3305 \\
\hline 0.04 & 0.03 & 0.03 & 0.035 & 2.1736 & 6.7290 & 8.0892 & 1.3078 & 0.3326 \\
\hline 0.02 & 0.02 & 0.01 & 0.02 & 2.3916 & 8.9410 & 6.3621 & 2.1046 & 0.2744 \\
\hline 0.02 & 0.02 & 0.02 & 0.03 & 2.2455 & 9.8846 & 6.8744 & 2.1239 & 0.3286 \\
\hline 0.02 & 0.02 & 0.03 & 0.035 & 1.8175 & 5.1596 & 6.5103 & 9.8878 & 0.3288 \\
\hline
\end{tabular}

Table 2: Values of $x_{1002}^{2}+y_{1002}^{2}, c_{20}+2 c_{11}+4 c_{02} \quad x_{1003}^{2}+y_{1003}^{2}, c_{20}+3 c_{11}+9 c_{02}$ and $3 \omega_{2}\left(x_{1003}^{2}+y_{1003}^{2}\right)^{1 / 2}$ for the Binary Systems

\begin{tabular}{|c|c|c|c|c|c|c|c|}
\hline $\begin{array}{l}\text { Binary } \\
\text { system }\end{array}$ & $\mathrm{A}_{1}$ & $\mathrm{~A}_{2}$ & $\begin{array}{l}\left(x_{1002}^{2}+y_{1002}^{2}\right) \\
\times\left(10^{3}\right)\end{array}$ & $\begin{array}{l}c_{20}+2 c_{11}+4 c_{02} \\
\times\left(10^{4}\right)\end{array}$ & $\begin{array}{l}x_{1003}^{2}+y_{1003}^{2} \\
\times\left(10^{7}\right)\end{array}$ & $\begin{array}{l}c_{20}+3 c_{11}+9 c_{02} \\
\times\left(10^{5}\right)\end{array}$ & $\begin{array}{l}3 \omega_{2}\left(x_{1003}^{2}+y_{1003}^{2}\right)^{1 / 2} \\
\times\left(10^{4}\right)\end{array}$ \\
\hline \multirow[t]{3}{*}{ Achird } & 0.01 & 0.02 & 1.8595 & 3.9506 & 4.6624 & 1.4198 & 0.2731 \\
\hline & 0.02 & 0.03 & 2.1416 & 7.3706 & 5.0439 & 1.5316 & 0.3268 \\
\hline & 0.03 & 0.035 & 1.8595 & 3.9506 & 7.1162 & 7.2991 & 0.3257 \\
\hline \multirow[t]{3}{*}{ Luyten } & 0.01 & 0.02 & 2.1181 & 5.9229 & 4.5846 & 1.3981 & 0.2730 \\
\hline & 0.02 & 0.03 & 2.0325 & 7.0807 & 4.9378 & 1.5108 & 0.3267 \\
\hline & 0.03 & 0.035 & 1.7759 & 3.7628 & 25.288 & 39.419 & 0.4297 \\
\hline \multirow[t]{3}{*}{$\alpha$ Cen $\mathrm{AB}$} & 0.01 & 0.02 & 1.8148 & 6.9036 & 4.1875 & 1.6006 & 0.2733 \\
\hline & 0.02 & 0.03 & 1.7160 & 7.7912 & 4.3966 & 1.6563 & 0.3270 \\
\hline & 0.03 & 0.035 & 1.4423 & 3.9703 & 5.2780 & 7.5691 & 0.3248 \\
\hline \multirow[t]{3}{*}{ Kruger-60 } & 0.01 & 0.02 & 2.1205 & 5.9310 & 4.5909 & 1.4000 & 0.2730 \\
\hline & 0.02 & 0.03 & 2.0348 & 7.0890 & 4.9466 & 1.5127 & 0.3268 \\
\hline & 0.03 & 0.05 & 1.7774 & 3.7678 & 7.0671 & 7.1981 & 0.3255 \\
\hline \multirow[t]{3}{*}{ Xi- Bootis } & 0.01 & 0.02 & 2.1632 & 6.0339 & 4.6994 & 1.4253 & 0.2731 \\
\hline & 0.02 & 0.03 & 2.0751 & 7.2018 & 5.0934 & 1.5376 & 0.3269 \\
\hline & 0.03 & 0.035 & 1.8081 & 3.8404 & 7.1639 & 7.3358 & 0.3258 \\
\hline
\end{tabular}

\section{Stability in resonance cases $\omega_{1}=3 \omega_{2}$}

In the resonance case $\omega_{1}=3 \omega_{2}$, it is possible to cancel whole of $\bar{H}_{3}$ of theHamiltonian $\bar{H}$ but ${ }^{4}$ retains the resonance terms and the terms with the same degree of canonical variables. Thus the normalized form of the Hamiltonian is written as:

$$
\begin{aligned}
& \bar{H}=i\left(\omega_{1} Q_{1} P_{1}+\omega_{2} Q_{2} P_{2}\right)-c_{20} Q_{1}^{2} P_{1}^{2}+ \\
& c_{11} Q_{1} P_{1} Q_{1} P_{2}-c_{02} Q_{2}^{2} P_{2}^{2}+h^{\prime}{ }_{1003} Q_{1} P_{2}^{3}+ \\
& h^{\prime}{ }_{0310} Q_{2}^{3} P_{1}+\ldots . .
\end{aligned}
$$

Again, applying the canonical transformation by means of polar coordinates given as:

$$
\begin{aligned}
Q_{1}^{0} & =\left(2 r_{i}\right)^{1 / 2} \sin \left(\varphi_{i}-\theta_{i}\right) \\
P_{1}^{0} & =\left(2 r_{i}\right)^{1 / 2} \cos \left(\varphi_{i}-\theta_{i}\right) ;(i=1,2)
\end{aligned}
$$

Where

$\theta_{2}=0$

$\sin \theta_{1}=\frac{x_{1003}}{\left(x_{1003}^{2}+y_{1003}^{2}\right)^{1 / 2}} ;$

$$
\cos \theta_{1}=\frac{-y_{1003}}{\left(x_{1003}^{2}+y_{1003}^{2}\right)^{1 / 2}} .
$$

The polar form of the normalized Hamiltonian reduces to the following form:

$$
\begin{aligned}
& \bar{H}=3 \omega_{2} r_{1}-\omega_{2} r_{2}+c_{20} r_{1}^{2}+c_{11} r_{1} r_{2}+c_{02} r_{2}^{2}+ \\
& \frac{\omega_{2}}{3} \times\left\{3\left(\sqrt{\left(x_{1003}^{2}+y_{1003}^{2}\right)}\right)\right\} \cdot r_{2}\left(r_{1} r_{2}\right)^{1 / 2} \\
& \sin \left(\varphi_{1}+3 \varphi_{2}\right)+o\left(\left(r_{1}+r_{2}\right)^{5 / 2}\right)
\end{aligned}
$$

Let us denote

$$
\begin{aligned}
& a=c_{20}+3 c_{11}+9 c_{02} ; \\
& d=3 \omega_{2}\left(x_{1003}^{2}+y_{1003}^{2}\right)^{1 / 2} .
\end{aligned}
$$

Now, according to Markeev theorem, if the following conditions are satisfied as:

i) $\left(x_{1003}^{2}+y_{1003}^{2}\right) \neq 0$ and $d \geq|a|$ holds simultaneously, then the equilibrium points are unstable and if $d<|a|$ then it is stable.

ii) When $\left(x_{1003}^{2}+y_{1003}^{2}\right)=0$ and $d=0$ are true simultaneously then also, it is stable.

iii) If $\left(x_{1003}^{2}+y_{1003}^{2}\right)=0$ and $d \neq 0$ then the stability in question is decided by the analysis of higher order terms in the normal form. The values of a and $d$ are calculated for various values of radiation pressure and oblateness parameter in table 1 .

From table (1) and (2) it is clear that for all the values of radiation pressures; $d<|a|$.Hence, the triangular points are stable in fourth order resonance for the binary systems.

\section{Discussion and conclusion}

The nonlinear stability of the triangular equilibrium points is examined in ER3BP in presence of resonance under the radiating and oblate primaries in circular case. The value of $\mu$ has been plotted for different values of radiation pressure and oblateness parameter. It is noted that under the range $\mu<\mu_{c}, \omega_{1}=\omega_{2}$ does not occur. It is clear for Fig1-3 that, for fixed value of radiation pres- 
sure and varying oblateness $\omega_{1}$ decreases with increasing $\mu$ whereas $\omega_{2}$ increases, and becomes equal for $\mu=\mu_{\mathrm{c}}$. The same behavior is seen for fixed oblateness but varying radiation pressure which is obvious from Figs.4-5. It is found from Figs 6-15 that the value of $\mu_{\mathrm{c} 1}$ and $\mu_{\mathrm{c} 2}$ increases or decreases with increasing value of oblateness parameter $A_{1}$ and $A_{2}$. From the above mentioned graph it is also clear that the values of $\mu_{\mathrm{c} 1}$ and $\mu_{\mathrm{c} 2}$ is less than the critical value $\mu_{\mathrm{c}}=0.0385209$ for all the values of radiation pressure and oblateness parameter taken, into consideration.

Here, by application of Markeev's theorems, it is found that for $\mu \leq \mu_{c}=0.0385209$; the motion is unstable for third order resonance but stable for fourth order resonance; for different values of radiation and oblateness parameter and for the binary systems (Achird, Luyten, $\alpha$ Cen AB, kruger-60, Xi- Bootis).

\section{References}

[1] Ammar MK (2008), the effect of solar radiation pressure on the Lagrangian points in the elliptical restricted three body problem, Astrophysics \& Space Science, 313, 393408.http://dx.doi.org/10.1007/s10509-007-9709-z.

[2] Arnold VI (1963a), Proof of A. N. Kolmogorov's theorem on the preservation of quasiperiodic motions under small perturbations of the Hamiltonian, Russian Math. Surveys, 18, 5, 9-36.

[3] Arnold VI (1963b), Small divisor problems in classical and celestial mechanics, Russian Mathematical Surveys 18(6), 85-192. http://dx.doi.org/10.1070/RM1963v018n06ABEH001143.

[4] Bennet A (1965), Characteristics exponents of the five equilibrium solutions in the elliptically restricted problem, Icarus 4, 177-187. http://dx.doi.org/10.1016/0019-1035(65)90060-6.

[5] Bhatnagar KB, Gupta U \&Bhadrawaj R (1994), Celestial Mechanics and Dynamical Astronomy, 59, 345-374. http://dx.doi.org/10.1007/BF00692102.

[6] Conxita P (1995), Ejection collision orbits with the more massive primary in the planar elliptic restricted three body problem, Celestial Mechanics and Dynamical Astronomy, 61, 315 331.http://dx.doi.org/10.1007/BF00049513.

[7] Danby, JMA (1964), Stability of the triangular points in the elliptic restricted problem of three bodies, Astronomical Journal, 69, 165-172. http://dx.doi.org/10.1086/109254.

[8] Erdi B (2009), A parametric study of stability and resonance around L4 in the elliptical restricted three body problem, Celestial Mechanics and Dynamical Astronomy, 104,145158.http://dx.doi.org/10.1007/s10569-009-9197-2

[9] Grebenikov EA (1964), On the stability of the Lagrangian Triangular Solutions of the Restricted Elliptic Three Body problem, Soviet Astronomy, 8(3), 451-459.

[10]Gyorgyey J (1985), on the nonlinear stability of motions around the elliptical restricted problem of three bodies, Celestial Mechanics and Dynamical Astronomy, 36(3), 281285.http://dx.doi.org/10.1007/BF01230741.

[11]Halan PP \& Rana N (2001), The Existence and stability of equilibrium points in the Robe's restricted three body problem, Celestial Mechanics and Dynamical Astronomy, 79, 145-155. http://dx.doi.org/10.1023/A:1011173320720.

[12] Kumar S \&Ishwar B (2011) Location of collinear equilibrium points in the generalized photogravitational elliptical restricted three body problem, Int. Journal of Sciience\& Technology, 3(2), 157-162.

[13]Kumar V \& Choudhary RK (1986), On the stability of the triangular libration points for the photogravitational circular restricted problem of three bodies when both of the attracting bodies are radiating as well, Celestial Mechanics, 40(2), 155 170.http://dx.doi.org/10.1007/BF01230257.

[14]Liapnuov AM (1956), the general problem of stability of Motion, Acad. Science USSR.

[15]Manju. \& Choudhary RK (1985), On the stability of triangular libration points taking into account the light pressure for the circular restricted problem of three bodies, Celestial Mechanics, 36, 165

[16]Markeev AP (1978), Libration points in celestial Mechanics and Cosmodynamics, nauka, Moscow, Russia.

[17]Markellos VV, Perdios E \&Labropoulou P (1992), Linear stability of the triangular equilibrium points in the phptogravitational elliptical restricted three body problem, Astrophysics \& Space Science, 194, 207 213. http://dx.doi.org/10.1007/BF00643991.

[18] Markellos VV (1996), Nonlinear stability zones around triangular equilibria in the plane circular restricted three-body problem , Astro- physics \& Space Science,

245

157.http://dx.doi.org/10.1007/BF00637811.

[19]Narayan A., and Shrivastava.A., (2014), Existence of Resonance Stability of Triangular Equilibrium Points in Circular Case of the Planar Elliptical Restricted Three-Body Problem under the Oblate and Radiating Primaries around the Binary System, Advances in Astronomy; http://dx.doi.org/10.1155/2014/287174.

[20]Narayan, A \&Singh.N (2014a), Motion and stability of triangular equilibrium points in elliptical restricted three body problem under the radiating primaries, Astrophysics \& Space Science, 352 (1) , 57-70. http://dx.doi.org/10.1007/s10509-014-1903-1.

[21] Narayan, A., Singh.N. (2014c), Resonance stability of triangular equilibrium points in elliptical restricted three body problem under the radiating primaries, Astrophysics \& Space Science, 353 (2) 441-455. http://dx.doi.org/10.1007/s10509-014-2085-6.

[22] Narayan, A \& Singh N (2014b), Stability of triangular lagrangian points in elliptical restricted three body problem under the radiating binary systems, Astrophysics \& Space Science, 353 (2) 457-464. http://dx.doi.org/10.1007/s10509-014-2014-8.

[23] Roberts G (2002), linear stability of the elliptic Lagrangian triangle solution in the three body problem, Differential equation, 182, 191218. http://dx.doi.org/10.1006/jdeq.2001.4089.

[24] Sahoo SK \&Ishwar B (2000), Stability of collinear equilibrium points in the generalized photo gravitational elliptic restricted three-body problem, Bulletin Astronomical Society India, 28, 579-586.

[25]Selaru D \&Cucu-Dumitrescu C (1995), Infinitesimal orbit around Lagrange points in the elliptic restricted three body problem , Celestial Mechanics and Dynamical Astronomy, 61(4), 333- 346. http://dx.doi.org/10.1007/BF00049514.

[26] Singh J \& Umar A (2012a), Motion in the photogravitational elliptic restricted three-body problem under an oblate primary, Astronomical Journal, 143, 109.http://dx.doi.org/10.1088/0004-6256/143/5/109.

[27] Singh J \& Umar A, (2012b), on the stability of triangular equilibrium points in the elliptic R3BP under radiating and oblate primaries, As$\begin{array}{llll}\text { trophysics } \& \text { Space } & \text { Science, }\end{array}$ 349.http://dx.doi.org/10.1007/s10509-012-1109-3.

[28] Szebebely V (1967), Stability of the points of equilibrium in the restricted problem, Astronomical Journal, 72, 7 9.http://dx.doi.org/10.1086/110195.

[29]Usha T, Narayan A \&Ishwar B (2014), Effects of Radiation and Triaxiality of primaries on triangular equilibrium points in elliptic restricted three body problem, Astrophysics \& Space Science, 349, 151-164. http://dx.doi.org/10.1007/s10509-013-1655-3.

[30]Zimvoschikov AS \&Thakai VN (2004), Instability of libration points and resonance phenomena in the photogravitational in the elliptical restricted three body problem, Solar System Research, 38 (2), 155163.http://dx.doi.org/10.1023/B:SOLS.0000022826.31475.a7.

\section{Appendices}

(a) The coefficient of third and fourth order terms of $h^{\prime} \alpha_{1} \alpha_{2} \beta_{1} \beta_{2}$ and $h_{\alpha_{1}} \alpha_{2} \beta_{1} \beta_{2}$ can be given as:

$h_{0030}=a_{1}^{3} b_{1}^{3} H_{0300}$

$\begin{aligned} h_{0030} & =a_{1}^{3}\left(H_{3000}+c_{1} H_{2100}+c_{1}^{2} H_{2100}\right. \\ & +c_{1}^{3} H_{0300} ;\end{aligned}$

$h_{1020}=a_{1}^{3} b_{1}^{2}\left(H_{1200}+3 c_{1} H_{0300}\right)$

$x_{1003}^{2}+y_{1003}^{2}$

$c_{20}+3 c_{11}+9 c_{02}$

$3 \omega_{2}\left(x_{1003}^{2}+y_{1003}^{2}\right)^{1 / 2}$

$h_{1001}=-2 a_{1}^{2} a_{2} b_{1} b_{2}\left(H_{1200}+3 c_{1} H_{0300}\right) ;$ 


$$
\begin{aligned}
& h_{1110}=2 a_{1}^{2} a_{2} b_{1}\left[H_{2100}+\left(c_{1}+c_{2}\right) H_{1200}\right. \\
& \left.+3 c_{1} c_{2} H_{0300}\right] \\
& h_{0021}=-3 a_{1}^{2} b_{1}^{2} b_{2} H_{0300} \text {; } \\
& h_{2100}=a_{1}^{2} a_{2}\left[3 H_{3000}+\left(2 c_{1}+c_{2}\right) H_{2100}+\right. \\
& \left.c_{1}\left(c_{1}+2 c_{2}\right) H_{1200}+3 c_{1}^{2} c_{2} H_{0300}\right] ; \\
& h_{1002}=a_{1} b_{1}^{2} a_{2}^{2}\left(H_{1200}+3 c_{1} H_{0300}\right) \text {; } \\
& h_{0210}=a_{1} a_{2}^{2} b_{1}\left(H_{2100}+2 c_{2} H_{1200}\right. \\
& \left.+3 c_{2}^{2} H_{0300}\right) \\
& h_{0012}=3 a_{1} a_{2}^{2} b_{2}^{2} b_{1} H_{0300} \\
& h_{1200}=a_{2}^{2} a_{1}\left[3 H_{3000}+\left(c_{1}+2 c_{2}\right) H_{2100}+\right. \\
& \left.c_{2}\left(2 c_{1}+c_{2}\right) H_{1200}+3 c_{1} c_{2}^{2} H_{0300}\right] \text {; } \\
& h_{0111}=-2 a^{2} a^{2} b_{b} b\left(H_{12}+3 c{ }^{H}{ }^{4}\right) \text {; } \\
& h_{1101}=-2 a_{2}^{2} a_{1} b_{2}\left[H_{2100}+\left(c_{1}+c_{2}\right) H_{1200}\right. \\
& \left.+3 c_{1} c_{2} H_{0300}\right] \\
& h_{0120}=a_{1}^{2} a_{2} b_{1}^{2}\left(H_{1200}+3 c_{2} H_{0300}\right) \text {, } \\
& h_{0201}=-a_{2}^{3} b_{2}\left(H_{2100}+2 c_{2} H_{1200}\right. \\
& \left.+3 c_{2}^{2} H_{0300}\right) \text {; } \\
& h_{0102}=a_{2}^{3} b_{2}{ }^{2}\left(H_{1200}+3 c_{2} H_{0300}\right) \text {; } \\
& h_{0003}=-a_{2}^{3} b_{2}{ }^{3} H_{0300} \text {; } \\
& h_{0300}=a_{2}^{3}\left(H_{3000}+c_{2} H_{2100}+c_{2}^{2} H_{1200}\right. \\
& \left.+c_{2}^{3} H_{0300}\right) \text {; } \\
& h_{0040}=a_{1}^{4} b_{1}{ }^{4} H_{0400} \text {; } \\
& h_{4000}=a_{1}^{4}\left(H_{4000}+c_{1} H_{3100}+c_{1}^{2} H_{2200}\right. \\
& \left.+c_{1}^{3} H_{1300}+c_{1}^{4} H_{0400}\right) \text {; } \\
& h_{2020}=a_{1}^{4} b_{1}^{2}\left(H_{2200}+3 c_{1} H_{1300}+6 c_{1}^{2} H_{0400}\right) ; \\
& h_{0022}=6 a_{1}^{2} a_{2}^{2} b_{1}^{2} b_{2}{ }^{2} H_{0400} \text {; } \\
& h_{2200}=a_{1}^{2} a_{2}{ }^{2}\left[6 H_{4000}+3\left(c_{1}+c_{2}\right) H_{3100}+\right. \\
& \left(c_{1}^{2}+4 c_{1} c_{2}+c_{2}^{2}\right) H_{2200}+ \\
& \left.3 c_{1} c_{2}\left(c_{1}+c_{2}\right) H_{1300}+6 c_{1}^{2} c_{2}^{2} H_{0400}\right] \text {; } \\
& \begin{aligned}
h_{2002}= & a_{1}^{2} a_{2}^{2} b_{2}^{2}\left(H_{2200}+3 c_{1} H_{1300}+\right. \\
& \left.6 c_{1}^{2} H_{0400}\right) ; \\
h_{0004}= & a_{2}^{4} b_{2}{ }^{4} H_{0400} ; \\
h_{0400}= & a_{2}^{4}\left(H_{4000}+c_{2} H_{3100}+c_{2}^{2} H_{2200}+c_{2}^{3} H_{1300}\right. \\
& \left.+c_{2}^{4} H_{0400}\right) ;
\end{aligned} \\
& h_{0202}=a_{2}^{4} b_{2}^{2}\left(H_{2200}+3 c_{2} H_{1300}+6 c_{2}^{2} H_{0400}\right) \\
& h_{0013}=-a_{1} a_{2}^{3} b_{2}{ }^{3} H_{0400} \\
& h_{1102}=a_{1} a_{2}{ }^{3}\left[4 H_{4000}+\left(c_{1}+3 c_{2}\right) H_{3100}+\right. \\
& 4 c_{1} c_{2}^{3} H_{0400}+2 c_{2}\left(c_{1}+c_{2}\right) H_{2200}+ \\
& \left.c_{2}^{2}\left(3 c_{1}+c_{2}\right) H_{1300}\right] \\
& h_{0211}=-2 a_{2}^{3} a_{1} b_{1} b_{2}\left[H_{2200}+3 c_{2} H_{1300}\right. \\
& \left.+6 c_{2}^{2} H_{0400}\right] \text {; } \\
& h_{0112}=3 a_{1} a_{2}^{3} b_{1} b_{2}^{2}\left(H_{1300}+4 c_{1} H_{0400}\right) ; \\
& h_{1003}=-a_{1} a_{2}^{3} b_{2}^{3}\left(H_{1300}+4 c_{1} H_{0400}\right) ; \\
& h_{1201}=-a_{1} a_{2}{ }^{3} b_{2}\left[3 H_{3100}+2\left(c_{1}+2 c_{2}\right) H_{2200}+\right. \\
& \left.3 c_{2}\left(2 c_{1}+c_{2}\right) H_{1300}+12 c_{1} c_{2}^{2} H_{0400}\right] \text {; } \\
& h_{0310}=a_{1} a_{2}^{3} b_{1}\left(H_{3100}+2 c_{2} H_{2200}+3 c_{2}^{2} H_{1300}\right. \\
& \left.+c_{2}^{2} H_{0400}\right) \text {; } \\
& { }_{0030}=h_{0030}-\frac{1}{\omega_{1}^{2}} h_{2010} ; \\
& y_{0030}=\frac{1}{\omega_{1}} h_{1020}-\frac{1}{\omega_{1}^{3}} h_{3000} ; \\
& x_{1020}=-\frac{1}{2} h_{1020}-\frac{3}{2 \omega_{1}^{2}} h_{3000} ; \\
& y_{1020}=\frac{3 \omega_{1}}{2} h_{0030}+\frac{1}{2 \omega_{1}} h_{2010} ; \\
& x_{0120}=-\frac{\omega_{2}}{2} h_{0021}+\frac{1}{2 \omega_{1}} h_{1110}+\frac{\omega_{2}}{2 \omega_{1}} h_{2001} ; \\
& y_{0120}=-\frac{1}{2} h_{0120}-\frac{\omega_{2}}{2 \omega_{1}} h_{1011}+\frac{1}{2 \omega_{1}^{2}} h_{2100} \\
& x_{1011}=-\omega_{1} h_{0021}-\frac{1}{\omega_{1}} h_{2001} ;
\end{aligned}
$$




$$
\begin{aligned}
& y_{0021}=h_{0021}+\frac{1}{\omega_{1} \omega_{2}} h_{1110}-\frac{1}{\omega_{1}^{2}} h_{2001} ; \\
& x_{1002}=-\frac{\omega_{1}}{2 \omega_{2}} h_{0111}-\frac{1}{2} h_{1002}+\frac{1}{2 \omega_{2}^{2}} h_{1200} ; \\
& y_{1002}=-\frac{\omega_{1}}{2} h_{0012}+\frac{\omega_{1}}{2 \omega_{1}^{2}} h_{0210}+\frac{1}{2 \omega_{2}^{2}} h_{1101} ; \\
& x_{0012}=-h_{0012}+\frac{1}{\omega_{2}^{2}} h_{0210}-\frac{1}{\omega_{1} \omega_{2}} h_{1101} ; \\
& y_{0012}=\frac{1}{\omega_{2}} h_{0111}-\frac{1}{\omega_{1}} h_{1002}+\frac{1}{\omega_{1} \omega_{2}^{2}} h_{1200} ; \\
& y_{0003}=-h_{0003}+\frac{1}{\omega_{2}^{2}} h_{0201} ; \\
& x_{0111}=\frac{\omega_{2}}{\omega_{1}} h_{1002}+\frac{1}{\omega_{1} \omega_{2}} h_{1200} ; y_{0111}=-\omega_{2} h_{0012}-\frac{1}{\omega_{2}} h_{0210} ; \\
& x_{0003}=\frac{-1}{\omega_{2}} h_{0102}+\frac{1}{\omega_{2}^{3}} h_{0300} ; \\
& x_{0201}=\frac{-\omega_{2}}{4} h_{0102}-\frac{3}{4 \omega_{2}} h_{0300} ; y_{0201}=\frac{-3 \omega_{2}^{2}}{4} h_{0003}+\frac{1}{4} h_{0201} ;
\end{aligned}
$$

(c)

$$
\begin{aligned}
& x_{1003}=\frac{1}{2} \omega_{1} h_{0013}+\frac{1}{2 \omega_{2}^{3}} h_{1300}-\frac{1}{2 \omega_{2}} h_{1102} \\
& -\frac{\omega_{1}}{2 \omega_{2}^{2}} h_{0211}-\frac{9}{5}\left(x_{0120^{x}} 0012+y_{0120} y_{0012}\right) \\
& -\frac{1}{\omega_{2}}\left(x_{1002}{ }_{1011}+x_{1011^{y}} 1002\right) \\
& +\frac{4}{\omega_{2}^{2}}\left(x_{1002^{x}} 0201+y_{1002}{ }^{y} 0201\right) \\
& -\frac{3}{2}\left(x_{0003^{x}} 0111+y_{0003^{y}} 0111\right) \text {; } \\
& y_{1003}=\frac{-1}{2 \omega_{2}} \omega_{1} h_{0112}-\frac{1}{2} h_{1003}+\frac{1}{2 \omega_{2}^{2}} h_{1201} \\
& +\frac{\omega_{1}}{2 \omega_{2}^{2}} h_{0310}-\frac{9}{5}\left(x_{0120} y_{0012}-y_{0120}{ }_{0012}\right) \\
& -\frac{1}{\omega_{2}}\left(y_{1002^{x}} 1011^{-x} 1011^{x} 1002\right) \\
& +\frac{4}{\omega_{2}^{2}}\left(y_{1002^{x} 0201}-x_{1002}{ }^{y} 0201\right) \\
& +\frac{3}{2}\left(y_{0003^{x}} 0111^{-x} 0003^{y} 0111\right) \text {. }
\end{aligned}
$$

